\title{
O TEOREMA DE BANACH-TARSKI NA CIRCUNFERÊNCIA
}

José Carlos de Oliveira Junior

Universidade Federal do Tocantins

Maria Cristina Cordeiro de Sousa

Universidade Federal do Tocantins cristinacordeiro18.to@uft.edu.br Nelly Almeida da Silva

Universidade Federal do Tocantins nellyalmeida@uft.edu.br

Matheus Pereira Lobo

Universidade Federal do Tocantins mplobo@uft.edu.br

\section{Resumo}

O Paradoxo de Banach-Tarski é um dos diversos paradoxos de decomposição [1]. Ele garante que é possível separar a circunferência $S^{1}$ em pedaços dois a dois disjuntos e, rotacionando-os adequadamente, formar duas novas circunferências. Neste trabalho, apresentamos este resultado e sua demonstração.

\section{Abstract}

The Banach-Tarski Paradox is one of several decomposition paradoxes [1]. It ensures that it is possible to disassemble the circumference $S^{1}$ into pairwise disjoint pieces and, by properly rotating them, reassemble into two new circumferences. In this paper, we present this result along with its proof.

\section{Introdução}

Os paradoxos de decomposição são frutos das propriedades dos conjuntos infinitos não-enumeráveis. O teorema de Banach-Tarski (BT), que é um paradoxo de decomposição, consiste em duplicar e ampliar esferas em $\mathbb{R}^{n}$. Outros paradoxos de decomposição fazem o mesmo para outros tipos de objetos [1]. Por meio de uma engenhosa sucessão de passos, é possível, por exemplo, particionar uma bola do tamanho de uma 
Oliveira Junior, J. C.; Cordeiro Sousa, M. C.; Almeida da Silva, N.; Pereira Lobo, M.

ervilha em finitos pedaços (isto é, encontrar um número finito de subconjuntos de uma bola de raio positivo pequeno) que podem ser rearranjados para formar uma bola do tamanho do Sol [2]! Esse resultado, associado com outras abordagens matemáticas, pode ter profundas implicações para a expansão do universo, levando-se em consideração, por exemplo, escalas microscópicas do espaço-tempo [3].

Ainda que o resultado seja conhecido, nossa abordagem, aqui, é original no sentido de que demonstramos o teorema na esfera unidimensional da forma mais simplificada que pudemos vislumbrar, utilizando, dentre outras ferramentas, rotações. Além disso, trazemos na Seção 4 algo mais surpreendente ainda, a saber, mostramos, sem artifícios de indução, que a circunferência unitária $S^{1}$ pode ser dividida em infinitas outras circunferências unitárias em um mesmo processo. As referências envolvendo a demonstração do Paradoxo de Banach-Tarski são demasiadamente técnicas [4, 5] e tratam em sua maioria de dimensões a partir de três. Um dos livros mais citados sobre decomposições paradoxais [1] está baseado em $\mathbb{R}^{3}$, e recomendamo-no fortemente àqueles que desejam aprofundar-se no tema.

O paradoxo de BT tornou-se a fundação para a teoria de medidas aditivas, que envolve a inter-relação entre análise (teoria da medida e funcionais lineares), álgebra (teoria combinatória de grupos), geometria (grupos de isometria) e topologia (grupos topológicos localmente compactos) [1]. Como veremos a seguir, utilizando classes de equivalência, enumerabilidade tanto dos racionais quanto dos subconjuntos infinitos dos naturais e, sobretudo, o axioma da escolha, é possível duplicar a circunferência $S^{1} \subset \mathbb{R}^{2}$. Mais especificamente, o resultado principal deste trabalho é o seguinte teorema.

Teorema 1.1. (Banach-Tarski) A circunferência $S^{1}$ pode ser particionada em um número infinito enumerável de subconjuntos de tal forma que, rotacionados convenientemente, pode-se gerar duas novas esferas $S^{1}$.

Destacamos que os axiomas de Zermelo-Fraenkel (ZF) são os axiomas padrão da teoria de conjuntos, e eles não incluem o axioma da escolha (AC, do inglês axiom of choice). O sistema ZF, que inclui AC, é denominado ZFC. O axioma da escolha é fundamental para o Paradoxo de Banach-Tarski. Por isso, ele não é um teorema em ZF. Em 1964, Solovay provou, a partir do método forcing de Paul Cohen, que ZF e "Não existe paradoxo de Banach-Tarski" são consistentes. Nas referências [1, 6], os leitores interessados poderão se aprofundar acerca do papel do axioma da escolha em paradoxos de decomposição [1] bem como compreender os processos matemáticos envolvidos no axioma da escolha [6]. 
Oliveira Junior, J. C.; Cordeiro Sousa, M. C.; Almeida da Silva, N.; Pereira Lobo, M.

\section{Preliminares}

Para apresentar a demonstração do teorema de Banach-Tarski, é necessário compreender alguns conceitos da Álgebra Abstrata, apresentados a seguir. Para uma revisão de Relações, consulte as referências [7, 8].

Definição 2.1. Seja $A$ um conjunto qualquer. Um conjunto $R \subseteq A \times A$ é chamado relação entre os elementos de $A$ ou, simplesmente, relação binária em $A$. Neste caso, dizemos que o elemento $x \in A$ está relacionado com o elemento $y \in A$ via relação $R$ se o par ordenado $(x, y) \in R$, e escreve-se $x R y$ ou $x \sim y$.

Neste trabalho, as relações de equivalência ganharão destaque e serão apresentadas na definição a seguir.

Definição 2.2. Seja $A$ um conjunto qualquer. Uma relação $R$ em $A$ é dita ser uma relação de equivalência se satisfaz essas três condições:

a. $\forall x \in A,(x, x) \in R$;

b. $\forall x, y \in A,(x, y) \in R \Rightarrow(y, x) \in R$;

c. $\forall x, y, z \in A,(x, y) \in R$ e $(y, z) \in R \Rightarrow(x, z) \in R$.

A condição $a$. é chamada de Reflexiva, b., Simétrica, e c., Transitiva. Essas condições serão úteis para a construção de um conjunto importante na demonstração do teorema de BT.

Existem várias aplicações e exemplos de relações de equivalência, e os interessados poderão encontrar alguns deles nas referências [7, 8].

Definição 2.3. Sejam $A$ um conjunto, $R \subseteq A \times A$ uma relação de equivalência em $A$ e $a \in A$. Definimos a classe de equivalência do elemento $a$ com relação a $R$ como:

$$
[a]=\{x \in A ;(x, a) \in R\} .
$$

Um exemplo famoso de classe de equivalência é o conjunto dos restos da divisão dos inteiros por $n \in \mathbb{N}=\{1,2,3, \cdots\}$.

O axioma a seguir tem em sua simplicidade um grau enorme de sofisticação. Decidimos apresentá-lo nesta seção, uma vez que ele será aplicado na demonstração do Teorema de BT em uma família de classes de equivalência.

Axioma 2.4. (Axioma da Escolha) Para qualquer conjunto de conjuntos não-vazios S, existe uma função chamada "função escolha para $S$ " tal que a imagem de cada conjunto $S$ é um elemento de $S[9,6]$. 
Oliveira Junior, J. C.; Cordeiro Sousa, M. C.; Almeida da Silva, N.; Pereira Lobo, M.

Dizer que é possível escolher um elemento de cada conjunto não-vazio de uma família de conjuntos é até aceitável quando esta família é infinita enumerável (tem a mesma cardinalidade dos naturais) ou finita. O que torna o Axioma da Escolha uma "verdade" intrigante é a possibilidade da família considerada de conjuntos não-vazios ser não enumerável (como, por exemplo, é o caso do conjunto dos números reais), o que acontece na demonstração do Teorema de BT.

A força do axioma da escolha está no fato de podermos demonstrar teoremas a partir da existência de uma função escolha, muito embora não possamos conhecê-la [10].

\section{Resultados Principais}

Antes de enunciarmos o Teorema de BT na circunferência, apresentamos sua versão em $\mathbb{R}^{3}$ cuja conclusão é completamente contra o senso comum [1].

Teorema 3.1. (Banach-Tarski em $\mathbb{R}^{3}$ ) Dada uma bola $E$ em $\mathbb{R}^{3}$, existe uma família finita de subconjuntos, dois a dois disjuntos, $A_{1}, A_{2}, \cdots, A_{n}, B_{1}, B_{2}, \cdots, B_{m}$ de $E$ e isometrias de $\mathbb{R}^{3} g_{1}, g_{2}, \cdots, g_{n}, h_{1}, h_{2}, \cdots, h_{m}$ tais que

$$
E=\bigcup_{i=1}^{n} g_{i}\left(A_{i}\right) \quad e \quad E=\bigcup_{j=1}^{m} h_{i}\left(B_{i}\right) .
$$

Nesse resultado, isometria significa, a grosso modo, movimentos no espaço $\mathbb{R}^{3}$ que não alteram o comprimento do vetor, ou seja, são rotações e translações. Em outras palavras, o Teorema 3.1 diz que toda bola em $\mathbb{R}^{3}$ pode ser dividida em um número finito de pedaços (subconjuntos da bola) tais que, reorganizando-os, é possível formar duas novas bolas iguais à primeira.

Na versão do Teorema de BT na circunferência, que enunciaremos e demonstraremos a seguir, ela será dividida em infinitos pedaços, dois a dois disjuntos, o que, apesar de diferir da versão em $\mathbb{R}^{3}$, continua sendo um resultado não intuitivo.

Teorema 3.2. (Banach-Tarski) A circunferência $S^{1}$ pode ser particionada em um número infinito enumerável de subconjuntos de tal forma que, rotacionados convenientemente, pode-se gerar duas novas esferas $S^{1}$.

Demonstração: Cada ponto da circunferência $S^{1}$, definida como sendo o conjunto dos pontos $(a, b)$ de $\mathbb{R}^{2}$ tais que $a^{2}+b^{2}=1$, está associado de maneira única a um ponto da forma $(\cos (\theta), \operatorname{sen}(\theta))$ para algum $\theta \in[0,2 \pi)$ em radianos, e a recíproca também é verdadeira. Para não carregarmos a notação e definirmos novas variáveis, vamos assumir, portanto, que cada $x \in S^{1}$ é um ângulo em radiano. Ainda a fim de simplificar a 
Oliveira Junior, J. C.; Cordeiro Sousa, M. C.; Almeida da Silva, N.; Pereira Lobo, M.

compreensão, não vamos fazer diferenciação entre pontos que são congruentes a outros módulo $2 \pi$, isto é, se $x, y \in S^{1}$ e $x=y+2 k \pi$ para algum $k \in \mathbb{Z}$, vamos dizer simplesmente que $x=y$. Isso faz sentido porque, quando olhamos os pontos $x$ e $y$ sobre a circunferência, não vemos dois pontos diferentes. Dito isso, considere a seguinte relação de equivalência em $S^{1}$, definida entre os ângulos $x$ e $y$ (em radianos) da circunferência:

$$
x \sim y \Leftrightarrow x-y \in \mathbb{Q} .
$$

Escrevemos $x-y \in \mathbb{Q}$ para representar que o ângulo $y$ é obtido a partir de $x$ por uma rotação de um ângulo racional. Esta relação na circunferência $S^{1}$ é de equivalência. Considere, então, $[x]=\left\{y \in S^{1} ; y \sim x\right\}$, isto é, $[x]$ é a classe de equivalência do elemento $x \in S^{1}$. Por estarmos tratando de uma relação de equivalência, é verdade que $x \in[x]$ seja qual for o elemento $x \in S^{1}$, pois a relação é reflexiva. Assim,

$$
S^{1}=\bigcup_{x \in S^{1}}[x]
$$

Em cada classe $[x]$, com $x \in S^{1}$, escolha apenas um elemento e considere $V$ o conjunto desses elementos, ou seja, $V=\left\{\right.$ um único elemento da classe $\left.[x] ; x \in S^{1}\right\}$. Isso é possível pelo Axioma da Escolha 2.4.

Seja $\left\{r_{i}\right\}_{i \in \mathbb{N}}$ uma enumeração do conjunto $\mathbb{Q}$ (veja [11]). Para cada $i \in \mathbb{N}$, considere

$$
V_{i}=r_{i}+V
$$

isto é, para cada $i \in \mathbb{N}$, o conjunto $V_{i}$ é a rotação de $V$ pelo ângulo $r_{i}$.

Afirmamos que

$$
S^{1}=\bigcup_{i=1}^{+\infty} V_{i}
$$

Com efeito, a inclusão $\bigcup_{i=1}^{+\infty} V_{i} \subseteq S^{1}$ é óbvia, pois os $V_{i}$ 's foram construídos a partir de elementos de $S^{1}$. Seja, então, $z \in S^{1}$ e considere sua classe [z]. Pela construção do conjunto $V$, existe $w \in V$ tal que $w \in[z]$. Pertencer à classe do elemento $z$ significa que a diferença $z-w$ é algum número racional, digamos $r_{i_{0}}$. Logo, $z=r_{i_{0}}+w \in r_{i_{0}}+V=V_{i_{0}}$, o que mostra a inclusão $S^{1} \subseteq \bigcup_{i=1}^{+\infty} V_{i}$ e prova nossa afirmação.

Sejam

$$
A=V_{1} \cup V_{3} \cup V_{5} \cup \cdots=\bigcup_{i=1}^{+\infty} V_{2 i-1}
$$

ReviSeM, Ano 2019, No. 2, 146-155 
Oliveira Junior, J. C.; Cordeiro Sousa, M. C.; Almeida da Silva, N.; Pereira Lobo, M.

e

$$
B=V_{2} \cup V_{4} \cup V_{6} \cup \cdots=\bigcup_{i=1}^{+\infty} V_{2 i} .
$$

Naturalmente, $S^{1}=A \cup B$. Pela construção de $V_{i}$, temos que $V_{i} \cap V_{j}=\emptyset$ para $i \neq j$, pois $V$ foi construído apenas com um único elemento de cada classe de equivalência. As classes de equivalência sempre formam uma partição, lembrando que a partição divide um conjunto em subconjuntos não-vazios e dois a dois disjuntos (veja [8]). Isso garante que $A \cap B=\emptyset$. Agora, vamos provar que é possível rotacionar os pontos dos conjuntos $A$ e $B$ e obter duas novas circunferências $S^{1}$.

Ora, podemos rotacionar $V_{2}$ para obtermos $V_{1}$. Basta rotacionarmos $V_{2}$ de um ângulo $r_{1}-r_{2}$. Teremos

$$
\left(r_{1}-r_{2}\right)+V_{2}=\left(r_{1}-r_{2}\right)+r_{2}+V=r_{1}+V=V_{1} .
$$

De modo geral, para cada $i \in \mathbb{N}$,

$$
\left(r_{i}-r_{2 i-1}\right)+V_{2 i-1}=\left(r_{i}-r_{2 i-1}\right)+r_{2 i-1}+V=r_{i}+V=V_{i}
$$

como também

$$
\left(r_{i}-r_{2 i}\right)+V_{2 i}=\left(r_{i}-r_{2 i}\right)+r_{2 i}+V=r_{i}+V=V_{i} .
$$

Utilizando esse processo em todos os elementos (pontos) de A e, similarmente, de B, segue de (3.1) que

$$
\begin{array}{ccccccccc}
V_{1} & \cup & V_{3} & \cup & V_{5} & \cup & \cdots & = & A \\
\downarrow & & \downarrow & & \downarrow & & \cdots & & \downarrow \\
V_{1} & \cup & V_{2} & \cup & V_{3} & \cup & \cdots & = & S^{1}
\end{array}
$$

como também

$$
\begin{array}{ccccccccc}
V_{2} & \cup & V_{4} & \cup & V_{6} & \cup & \cdots & = & B \\
\downarrow & & \downarrow & & \downarrow & & \cdots & & \downarrow \\
V_{1} & \cup & V_{2} & \cup & V_{3} & \cup & \cdots & = & S^{1} .
\end{array}
$$

Portanto, uma vez que $S^{1}=A \cup B$, rotacionando os pontos dos conjuntos disjuntos $A$ e $B$, obtemos duas novas circunferências $S^{1}$, o que conclui a prova do teorema.

\section{Comentários}

Uma pergunta pertinente seria: É possível separar a circunferência $S^{1}$ em pedaços que, após rotações, seja possível obter três novas circunferências $S^{1}$ ? E quatro novas 
Oliveira Junior, J. C.; Cordeiro Sousa, M. C.; Almeida da Silva, N.; Pereira Lobo, M.

circunferências? E infinitas? A resposta para todas essas perguntas é positiva. A seguir, mostramos como se procede para responder cada uma.

Para obtermos três novas circunferências, precisamos separar apropriadamente os índices dos $V_{i}^{\prime}$ 's da união (3.1) dada por $S^{1}=\bigcup_{i=1}^{+\infty} V_{i}$. Considere, para isso, $A=\bigcup_{i=1}^{+\infty} V_{3 i}$, $B=\bigcup_{i=1}^{+\infty} V_{3 i+1}$ e $C=\bigcup_{i=1}^{+\infty} V_{3 i+2}$. Note que $A, B$ e $C$ são dois a dois disjuntos (uma vez que os próprios $V_{i}$ 's o são) e também $A=V_{3} \cup V_{6} \cup V_{9} \cup \cdots, B=V_{4} \cup V_{7} \cup V_{10} \cup \cdots$. e $C=V_{5} \cup V_{8} \cup V_{11} \cup \cdots$. Agora, procedemos da mesma forma que anteriormente, rotacionando cada $V_{3 i}$ em $A$, cada $V_{3 i+1}$ em $B$ e cada $V_{3 i+2}$ em $C$ para obtermos três novas circunferências. $\mathrm{O}$ caso de quatro novas circunferências é análogo, bastando considerar os conjuntos $A=\bigcup_{i=1}^{+\infty} V_{4 i}, B=\bigcup_{i=1}^{+\infty} V_{4 i+1}, C=\bigcup_{i=1}^{+\infty} V_{4 i+2}$ e $D=\bigcup_{i=1}^{+\infty} V_{4 i+3}$. Para se obter infinitas novas circunferências, seja $\left\{p_{1}, p_{2}, p_{3}, \cdots\right\}$ uma enumeração dos números primos positivos. Considere, para cada $i \in \mathbb{N}=\{1,2,3, \cdots\}$, o conjunto

$$
X_{i}=\left\{p_{i}^{k} ; k \in \mathbb{N}\right\}
$$

isto é, o conjunto $X_{i}$ é formado por todas as potências do número $p_{i}$. Seja $X_{0}$ o conjunto dos números naturais que não são potências de nenhum primo. Dessas considerações, temos que $\mathbb{N}=X_{0} \cup X_{1} \cup X_{2} \cup \cdots$, em que a união é formada por conjuntos dois a dois disjuntos, e cada $X_{i}$ possui infinitos elementos. Agora, vamos construir os pedaços da circunferência que servirão para gerar outras circunferências. Seja, para cada $i \in$ $\mathbb{N} \cup\{0\}$, o conjunto

$$
A_{i}=\bigcup_{j \in X_{i}} V_{j}
$$

Por exemplo, considerando $p_{1}=2$ e $p_{2}=3$, temos que $A_{1}=V_{2} \cup V_{4} \cup V_{8} \cup V_{16} \cup \cdots$ e $A_{2}=V_{3} \cup V_{9} \cup V_{27} \cup V_{81} \cup \cdots$. Ora, procedendo, então, como antes, é possível tornar cada pedaço $A_{i}$ de $S^{1}$ em uma nova circunferência $S^{1}$.

Por fim, apresentamos a Figura 1, que é uma representação ilustrativa do Teorema de Banach-Tarski. 
Oliveira Junior, J. C.; Cordeiro Sousa, M. C.; Almeida da Silva, N.; Pereira Lobo, M.

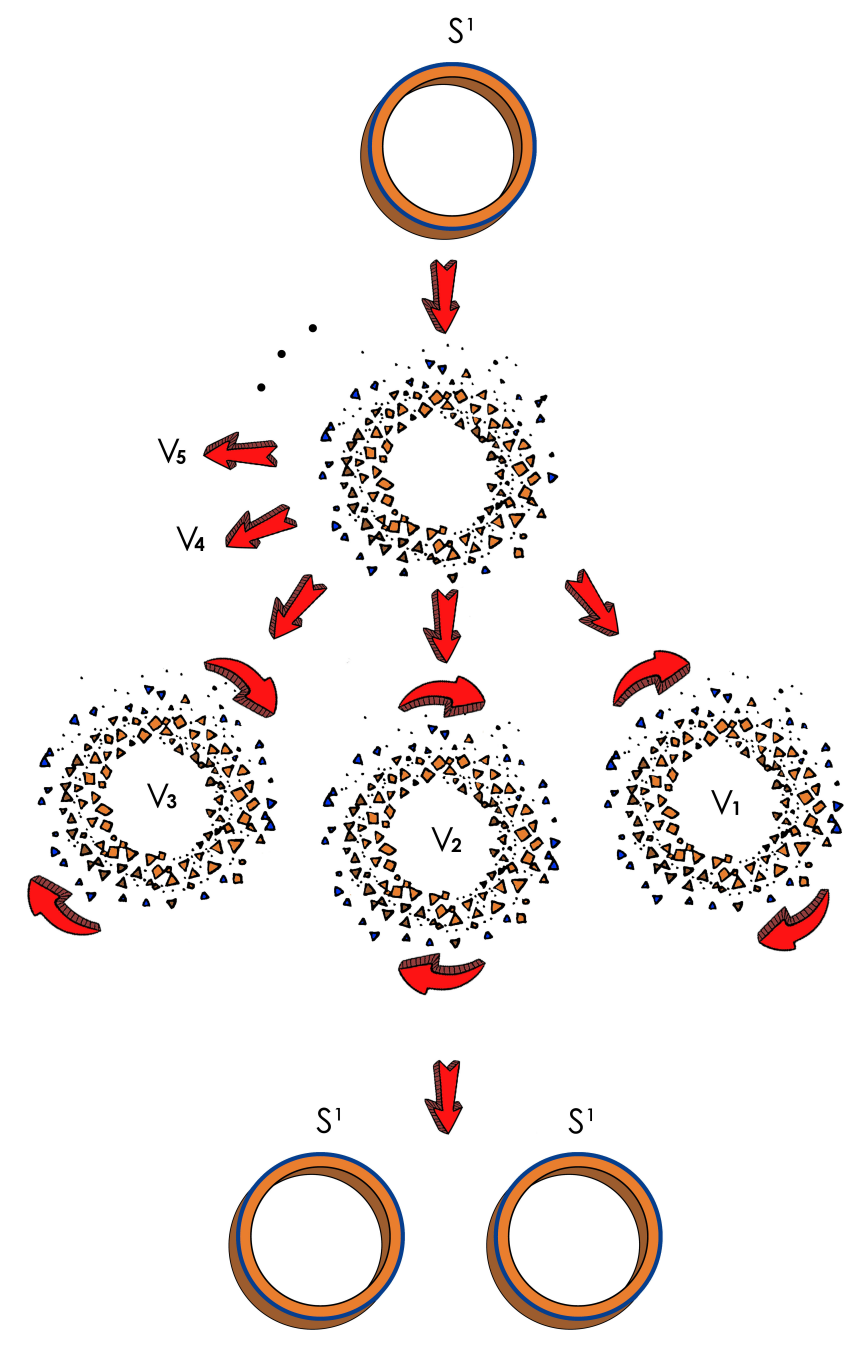

Figura 1: Ilustração do Teorema de Banach-Tarski. 
Oliveira Junior, J. C.; Cordeiro Sousa, M. C.; Almeida da Silva, N.; Pereira Lobo, M.

\section{Considerações Finais}

O Paradoxo de Banach-Tarski é intrigante em sua essência. Demonstramos o teorema na circunferência $S^{1}$ utilizando relações, classes de equivalência, rotações, axioma da escolha, enumeração e a bijeção entre os números pares e ímpares e o conjunto dos Naturais. Além de duplicar a circunferência, mostramos que é possível triplicar, quadruplicar e, até mesmo, dividi-la em $k$ novas circunferências seja qual for o número natural $k$. Ademais, provamos ainda que é possível dividir a circunferência em infinitas outras circunferências. A validade deste teorema reside em diversas partes, valendo a pena destacar o axioma da escolha, as bijeções utilizadas e partições a partir de classes de equivalência. Esses itens foram fundamentais para a demonstração apresentada.

Como sugestão para futuros trabalhos, destacamos a duplicação da esfera $S^{2}$ bem como o estudo da unicidade da medida de Lebesgue nos conjuntos envolvidos em Paradoxos de Banach-Tarski [1].

\section{Agradecimentos}

Agradecemos à Universidade Federal do Tocantins por oferecer a infraestrutura necessária para a realização deste trabalho.

\section{Referências}

[1] G. Tomkowicz, S. Wagon, The Banach-Tarski Paradox, 2nd ed., Cambridge University Press, 2016.

[2] L.M. Wapner. The Pea and the Sun: a mathematical paradox, AK Peters/CRC Press, 2005.

[3] M.S. El Naschie, Banach-Tarski theorem and Cantorian micro space-time. Chaos, Solitons \& Fractals 5, 1503-1508 (1995).

[4] K. Stromberg, The Banach-Tarski paradox, The American Mathematical Monthly 86, 151-161 (1979).

[5] M. Laczkovich, Paradoxical decompositions: a survey of recent results, First European Congress of Mathematics, Paris, July 6-10 (1992).

[6] H. Herrlich, Axiom of choice, Berlin: Springer, 2006. 
Oliveira Junior, J. C.; Cordeiro Sousa, M. C.; Almeida da Silva, N.; Pereira Lobo, M.

[7] G. Iezzi e C. Murakami, Fundamentos de Matemática Elementar: Conjuntos e Funções, 3 ed., Atual, 1977.

[8] D. J. Velleman, How To Prove It: a structured approach, 2nd ed., Cambridge University Press, 2006.

[9] J. Stillwell, Roads to Infinity: the mathematics of truth and proof, CRC Press, 2010.

[10] S. Warner, Set Theory for Beginners: a rigorous introduction to sets, relations, partitions, functions, induction, ordinals, cardinals, Martin's axiom and stationary sets, Get 800, 2019.

[11] E. L. Lima, Curso de Análise, Vol. 1, IMPA, 2017.

Submetido em 22 de Dezembro de 2018. Aceito em 01 de Junho de 2019. 